\title{
Gradient Competition Anisotropy for Centerline Extraction and Segmentation of Spinal Cords
}

\author{
Max W.K. Law ${ }^{1,2, \star}$, Gregory J. Garvin ${ }^{2,4}$, Sudhakar Tummala ${ }^{2}$, \\ KengYeow Tay ${ }^{2,3}$, Andrew E. Leung ${ }^{2,3}$, and Shuo $\mathrm{Li}^{1,2}$ \\ ${ }^{1}$ GE Healthcare, Canada \\ ${ }^{2}$ University of Western Ontario, London, Canada \\ ${ }^{3}$ London Health Sciences Centre, London, Canada \\ ${ }^{4}$ St. Joseph's Health Care London, London, Canada \\ max.w.k.law@gmail.com
}

\begin{abstract}
Centerline extraction and segmentation of the spinal cord - an intensity varying and elliptical curvilinear structure under strong neighboring disturbance are extremely challenging. This study proposes the gradient competition anisotropy technique to perform spinal cord centerline extraction and segmentation. The contribution of the proposed method is threefold - 1) The gradient competition descriptor compares the image gradient obtained at different detection scales to suppress neighboring disturbance. It reliably recognizes the curvilinearity and orientations of elliptical curvilinear objects. 2) The orientation coherence anisotropy analyzes the detection responses offered by the gradient competition descriptor. It enforces structure orientation consistency to sustain strong disturbance introduced by high contrast neighboring objects to perform centerline extraction. 3) The intensity coherence segmentation quantifies the intensity difference between the centerline and the voxels in the vicinity of the centerline. It effectively removes the object intensity variation along the structure to accurately delineate the target structure. They constitute the gradient competition anisotropy method which can robustly and accurately detect the centerline and boundary of the spinal cord. It is validated and compared using 25 clinical datasets. It is demonstrated that the proposed method well suits the applications of spinal cord centerline extraction and segmentation.
\end{abstract}

\section{Introduction}

Centerline and boundary detection of the spinal cord in T1-weighted (T1-) and T2-weighted (T2-) magnetic resonance (MR) images are beneficial to the diagnosis of spinal cord compression, myelopathy, spinal cord atrophy and multiple sclerosis. In T1- and T2-MR images, a spinal cord is a grey elliptical curvilinear object surrounded by different tissues, including dark bone cortex/spinal cavity/cartilaginous tissues, dark to grey ligaments and nerve roots, grey bone marrow and dark grey to bright cerebro-spinal fluid (CSF). The intensity of the

\footnotetext{
* Corresponding author.
} 
spinal cord and the surrounding tissues can noticeably change because of the reduced amount of white matter of the spinal cord towards the inferior direction, fluid motion artifacts, partial volume effects and bias fields. Considering the strong disturbance introduced by adjacent tissues (Figs. 1 $1 \mathrm{~h}-\mathrm{f}$ ) and the inconsistent intensity of the spinal cord, the detection of the spinal cord centerline and boundary are extremely challenging tasks.

Prior curvilinear structure detection approaches [1 [9] mainly focus on detection of circular tubular objects. They scarcely discuss the analysis of elliptical curvilinear structures surrounded by high contrast neighboring objects. Owing to the lack of a suitable curvilinear structure descriptor, previous spinal cord detection approaches rely on extensive user inputs, training data and prior assumption of the spinal cord. Koh et. al. [10] segmented the surrounding structures of a spinal cord to include the spinal cord in the segmentation result. An atlas-based non-rigid registration algorithm was proposed for voxel-wise classification for spinal cord segmentation [11]. McIntosh and Harmarneh [12] designed a set of criteria including structure surface smoothness, structure cross section circularity and intensity consistency, along with user guided centerline extraction to segment the spinal cord. Horsfield et. al. [13] formulated an active surface model for spinal cord segmentation which requires manually supplied spinal cord centerlines. Coulon et. al. 14] presented a gradient based active surface model which merely refines manually segmented spinal cord surfaces.

This paper proposes the gradient competition anisotropy to detect elliptical curvilinear structure under strong neighboring disturbance. The proposed method possesses three components - 1) the gradient competition descriptor sustains neighboring disturbance to detect elliptical curvilinear objects; 2) the orientation coherence anisotropy reliably extracts structure centerlines even if high contrast neighboring objects present; 3) the intensity coherence segmentation well handles the intensity inconsistency along the structure.

The gradient competition descriptor captures the structure orientation and curvilinearity. The curvilinearity and orientation, along with the orientation coherence constitute a Riemannian metric for orientation coherence anisotropy centerline extraction. Based on minimizing the intensity difference between the centerline and the voxels in the vicinity of the centerline, the spinal cord is delineated by the intensity coherence segmentation. Distinct from the aforementioned existing spinal cord detection approaches, the proposed method is general to the

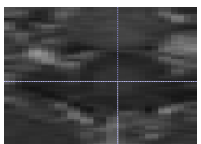

(a)

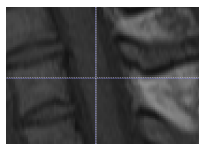

(b)

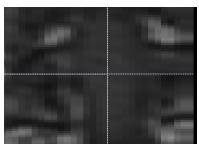

(c)

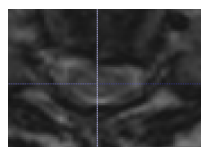

(d)

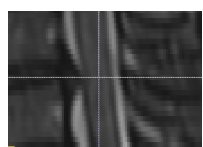

(e)

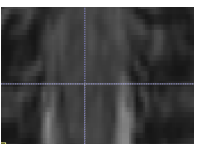

(f)

Fig. 1. Local image patches of two volumetric cervical spinal scan. (a-c) T1-MR image, the crosses belong to a manually selected center of the spinal cord; (d-f) T2-MR image, the crosses belong to a manually selected center of the spinal cord. (a,d) Axial planes; (b, e) Sagittal planes; (c, f) Coronal planes. 
detection of different curvilinear structures. It also handles both T1- and T2MR images. It is validated and compared using $10 \mathrm{~T} 1-$ and $15 \mathrm{~T} 2-\mathrm{MR}$ images in the experiment. It is shown that the proposed method well suits the tasks of elliptical curvilinear structure detection under strong neighboring disturbance, so as the spinal cord centerline extraction and segmentation.

\section{Methodology}

\subsection{Gradient Competition Descriptor}

The anisotropic descriptor is developed based on aggregating image gradients at a spherical surface $\delta S_{r}$,

$$
f(\boldsymbol{x} ; r, \hat{\rho})=\frac{1}{4 \pi r^{2}} \int_{\delta S_{r}}\left(\left(\hat{\rho} \hat{\rho}^{T}\right) \nabla I(\boldsymbol{x}+r \hat{n})\right) \cdot \hat{n} d A,
$$

where $r$ is the radius of the spherical surface, $\hat{\rho}$ is an arbitrary detection direction, $\hat{n}$ and $d A$ are the outward normal and infinitesimal area of the spherical surface respectively. Derivatives are evaluated by convolving images with the first derivatives of Gaussian having a unit voxel-length scale factor. The term $\hat{\rho} \hat{\rho}^{T} \nabla I$ reports the gradient along $\hat{\rho}$. This descriptor reports positive (negative) responses when $\delta S_{r}$ touches an ascending (descending) edge [6, 2].

Inside a curvilinear structure, the optimal detection direction which produces the strongest response lies on the structure cross-sectional plane. If $\hat{\rho}$ is parallel to the curvilinear structure orientation, the response is minimal. Given the optimal detection direction, the above measure reaches its maximum at the curvilinear object centerline when $r$ is the semi-width of the object, i.e. the contact area between $\delta S_{r}$ and the curvilinear object boundary is maximized. The optimal detection direction is obtained according to,

$$
\hat{a}_{1, r}=\arg \max _{\hat{\rho}}|f(\boldsymbol{x} ; r, \hat{\rho})| \underline{1}
$$

The response $f\left(\cdot ; q, \hat{a}_{1, q}\right)$ is competed with the strongest responses reported in a smaller detection scale to penalize oversize detection scales,

$$
\lambda_{1, r}=\max \left(f\left(\cdot ; r, \hat{a}_{1, r}\right), 0\right)-\max _{u \in\{l, 2 l, \ldots, r\}}\left|f\left(\cdot ; u, \hat{a}_{1, r}\right)\right|,
$$

where $l$ is the minimum voxel length. The above equation assumes that the target curvilinear object is darker than its surrounding (spinal cords in T2MR images). Detection of bright structure (spinal cords in T1-MR images) is achieved by conducting the analysis on the inverted image. On spinal cord detection, the above gradient competition suppresses the disturbance introduced by

${ }^{1}$ The optimization of Equations 2 and 5 are achieved by performing eigendecomposition on a 3-by-3 symmetric tensor $\left(4 \pi r^{2}\right)^{-1} \int_{S_{r}} \mathbf{H} d V$ and a 2-by-2 symmetric tensor $\left(4 \pi r^{2}\right)^{-1} \int_{S_{r}}\left[\frac{\partial}{\partial \hat{b}_{1}} \frac{\partial}{\partial \hat{b}_{2}}\right]\left[\frac{\partial}{\partial \hat{b}_{1}} \frac{\partial}{\partial \hat{b}_{2}}\right]^{T}(I) d V$ respectively, where $\mathbf{H}$ is the image Hessian, $\hat{b}_{1} \times \hat{b}_{2}=\hat{b}_{1} \times \hat{a}_{1, r_{1}}=\hat{b}_{2} \times \hat{a}_{1, r_{1}}=0$ and $V$ is the infinitesimal volume. 
neighboring structures, such as CSF, ligaments and bone cortex when extracting responses inside the spinal cord. The optimal detection scale is,

$$
r_{1}=\arg \max _{r \in\left\{Z_{0}, Z_{0}+l, \ldots Z_{1}\right\}} \lambda_{1, r}
$$

where $Z_{0}$ and $Z_{1}$ are the minimum and maximum possible semi-widths of the spinal cord respectively 2 . To conform with the elliptical curvilinear object, a different detection scale is obtained in the orthogonal space of $\hat{a}_{1, r_{1}}$ [15],

$$
\begin{aligned}
\hat{a}_{2, r} & =\arg \max _{\hat{\rho}, \forall \hat{\rho} \perp \hat{a}_{1, r 1}}|f(\cdot ; r, \hat{\rho})|,^{1} \\
\lambda_{2, r} & =\max \left(f\left(\cdot ; r, \hat{a}_{2, r}\right), 0\right)-\max _{u \in\{l, 2 l, \ldots, r\}}\left|f\left(\cdot ; u, \hat{a}_{2, r}\right)\right|, r_{2}=\underset{r \in\left\{Z_{0}, Z_{0}+l, \ldots Z_{1}\right\}}{\arg \max } \lambda_{2, r} .
\end{aligned}
$$

A curvilinear structure orientation field is retrieved as $\hat{v}=\hat{a}_{1, r_{1}} \times \hat{a}_{2, r_{2}}$. The object curvilinearity is defined as the sum of the intensity changes cross the boundaries, subtracted by the local intensity fluctuation along the structure,

$$
\gamma=\lambda_{1, r_{1}}+\lambda_{2, r_{2}}-\max _{r \in\left[l, 2 l, \ldots Z_{1}\right]}|f(\cdot ; r, \hat{v})|
$$

\subsection{Orientation Coherence Anisotropy}

The gradient competition descriptor offers a scalar curvilinearity response $\gamma$ and an orientation field $\hat{v}$. The spinal cord centerline has both large curvilinearity and low orientation discrepancy. An anisotropic speed functional is constructed to capture the spinal cord centerline,

$$
\arg \max _{\mathcal{P} \in \mathbf{P}_{\boldsymbol{p}_{0}, \boldsymbol{p}_{1}}} \int_{\mathcal{P}}\left(\gamma(\mathcal{P}(p))\left|\hat{v}(\mathcal{P}(p)) \cdot \mathcal{P}^{\prime}(p)\right|\right) d p,
$$

where $p$ is the length parameterization of the path $\mathcal{P}, \mathbf{P}_{\boldsymbol{p}_{0}, \boldsymbol{p}_{1}}$ is a family of paths which connect the points $\boldsymbol{p}_{0}$ and $\boldsymbol{p}_{1} \cdot \boldsymbol{p}_{0}$ and $\boldsymbol{p}_{1}$ are the manually supplied end points of the spinal cord. Equation 8 finds the fastest pathway to travel from $\boldsymbol{p}_{0}$ to $\boldsymbol{p}_{1}$ to extract the spinal cord centerline.

However, the structure orientation field can be adversely affected by noise or ambiguous object boundaries. Computation of the optimal path can be deteriorated by the inaccurately estimated structure orientation, leading to an incorrect path which follows the large curvilinearity responses induced by adjacent high contrast objects. This is overcome by considering the orientation coherence between the path and the structure orientation in a local region,

$$
\begin{aligned}
& \arg \max _{\mathcal{P} \in \mathbf{P}_{\boldsymbol{p}_{0}, \boldsymbol{p}_{1}}} \int_{\mathcal{P}} J_{\text {Speed }}\left(p, \mathcal{P}, \mathcal{P}^{\prime}\right) d p, \text { where } \\
& J_{\text {Speed }}\left(p, \mathcal{P}, \mathcal{P}^{\prime}\right)=\gamma(\mathcal{P}(p))\left(\int g_{\sigma}(\boldsymbol{y})\left(\hat{v}(\mathcal{P}(p)+\boldsymbol{y}) \cdot \mathcal{P}^{\prime}(p)\right)^{2} d V\right)^{\frac{1}{2}} .
\end{aligned}
$$

\footnotetext{
${ }^{2} Z_{0}$ and $Z_{1}$ are $1.5 \mathrm{~mm}$ and $20 \mathrm{~mm}$ in this study.
} 
where $g_{\sigma}$ is a Gaussian function with a scale factor $\sigma, d V$ is the infinitesimal volume. Given $\sigma$ is defined according to the minimum width of the detection target 3 , the structure orientation at the spinal cord centerline is coherent within the effective Gaussian window, i.e. $\left(\int g_{\sigma}(\boldsymbol{y})\left(\hat{v}(\mathcal{P}(p)+\boldsymbol{y}) \cdot \mathcal{P}^{\prime}(p)\right)^{2} d V\right)^{\frac{1}{2}} \approx$ $\left|\hat{v}(\mathcal{P}(p)) \cdot \mathcal{P}^{\prime}(p)\right|$ and Equation 9 resembles Equation 8 , When the object orientation becomes ambiguous, the locally random orientation field lowers the preference of the optimal path orientation. It is distinct to Equation 8 which always forces the path to follow the orientation field. Thus, the optimal path extraction is more robust against less accurate orientation fields caused by noise or ambiguous object boundaries. Equation 9 is reformulated in a tensorial form to compute the corresponding optimal path, $J_{\text {Speed }}\left(p, \mathcal{P}, \mathcal{P}^{\prime}\right)$ becomes,

$$
\begin{aligned}
& \left(\gamma^{2}(\mathcal{P}(p))\left(\int g_{\sigma}(\boldsymbol{y})\left(\mathcal{P}^{\prime}(p)\right)^{T}\left(\hat{v}(\mathcal{P}(p)+\boldsymbol{y}) \hat{v}^{T}(\mathcal{P}(p)+\boldsymbol{y})\right) \mathcal{P}^{\prime}(p) d V\right)\right)^{\frac{1}{2}} \\
= & \sqrt{\left(\mathcal{P}^{\prime}(p)\right)^{T}\left(\int \gamma^{2}(\mathcal{P}(p)) g_{\sigma}(\boldsymbol{y}) \hat{v}(\mathcal{P}(p)+\boldsymbol{y}) \hat{v}^{T}(\mathcal{P}(p)+\boldsymbol{y}) d V\right) \mathcal{P}^{\prime}(p)} .
\end{aligned}
$$

Let $\mathbf{M}$ denote the resultant tensors of integrating the orientation field outer product in Equation 10. The computation of $\mathbf{M}$ is independent to the path $\mathcal{P}$,

$$
\mathbf{M}(\boldsymbol{x})=\gamma^{2}(\boldsymbol{x})\left(\int g_{\sigma}(\boldsymbol{y}) \hat{v}(\boldsymbol{x}+\boldsymbol{y}) \hat{v}^{T}(\boldsymbol{x}+\boldsymbol{y}) d V\right) .
$$

$\mathbf{M}$ can be regarded as a tensor defining an anisotropic local speed. It embodies the curvilinearity and orientation field provided by the gradient competition descriptor. A minimum time of arrival function $\mathcal{T}(\boldsymbol{x})$ is defined as,

$$
\mathcal{T}(\boldsymbol{x})=\min _{\mathcal{P} \in \mathbf{P}_{p_{0}, x}} \int_{\mathcal{P}} J\left(p, \mathcal{P}, \mathcal{P}^{\prime}\right) d p, J\left(p, \mathcal{P}, \mathcal{P}^{\prime}\right)=\sqrt{\mathcal{P}^{\prime}(p)^{T} \mathbf{N}^{-1}(\mathcal{P}(p)) \mathcal{P}^{\prime}(p)},
$$

where $\mathcal{T}\left(\boldsymbol{p}_{0}\right)=0, \mathbf{N}=\mathbf{M}+\epsilon \mathbf{I}, \epsilon$ is a small constant ( $10^{-4}$ in this paper) to avoid singularity and $\mathbf{I}$ is an identity matrix. The optimal path is acquired using gradient descent according to $\mathcal{L}^{\prime} \propto \mathbf{N} \nabla \mathcal{T}$. The first variation of $\mathcal{T}$ is,

$$
\delta \mathcal{T}=\frac{\partial J}{\partial \mathcal{P}^{\prime}} \delta \mathcal{P}+\int_{p 0}^{x}\left(\frac{\partial J}{\partial \mathcal{P}}-\frac{d}{d s} \frac{\partial J}{\partial \mathcal{P}^{\prime}}\right) d p .
$$

The second term on the right hand side of the above equation vanishes according to the Euler condition, i.e.

$$
\nabla \mathcal{T}=\frac{\partial \mathcal{T}}{\partial \mathcal{P}}=\frac{\partial J}{\partial \mathcal{P}^{\prime}}=\frac{\mathbf{N}^{-1} \mathcal{P}^{\prime}}{\sqrt{\mathcal{P}^{\prime T} \mathbf{N}^{-1} \mathcal{P}^{\prime}}}
$$

It can be expressed in a form of Eikonal equation,

$$
\nabla \mathcal{T}^{T} \mathbf{N} \nabla \mathcal{T}=\frac{\mathcal{P}^{\prime T}\left(\mathbf{N}^{-1}\right)^{T} \mathbf{N N}^{-1} \mathcal{P}^{\prime}}{\mathcal{P}^{\prime T} \mathbf{N}^{-1} \mathcal{P}^{\prime}}=1
$$

The Eikonal equation is solved by the anisotropic fast marching algorithm [16].

3 The full width of half maximum of the Gaussian function is decided according to the minimum width of the spinal cord, i.e. $\sigma=0.5(2 \ln 2)^{-0.5} Z_{0}$. 
The optimal path of Equation 12 is acquired based on the minimal Riemannian arc-length governed by the tensorial metric $\mathbf{M}^{-1}$. When the local orientation is coherent, $\mathbf{M}$ is a stick tensor, giving a $\gamma^{2}$ and null speed along the structure orientation and structure cross-sectional plane respectively. Such a tensor yields an infinite anisotropy, leading to a strong preference to align the optimal path along the structure orientation. If the orientation is locally random, $\mathbf{M}$ becomes a ball-tensor, possessing unit anisotropy and a $\frac{1}{3} \gamma^{2}$ isotropic speed. The reduced speed $\frac{1}{3} \gamma^{2}$ leads to a more conservative path search, while the tensor has a minimal anisotropy, implying a less restrictive path orientation preference. As such, the tensor anisotropy is governed by the local orientation field coherence. It effectively avoids the optimal path from following a random orientation field and producing incorrect centerline extraction.

\subsection{Intensity Coherence Segmentation}

Let $\boldsymbol{y}_{\boldsymbol{x}}$ denotes a centerline position which is closest to the voxel $\boldsymbol{x}$. An intensity difference function is formulated,

$$
\mathcal{E}=\left|I(\boldsymbol{x})-I\left(\boldsymbol{y}_{\boldsymbol{x}}\right)\right|
$$

The spinal cord is segmented by partitioning the vicinity of the centerline into two regions. The desired region is the one connecting to the centerline with the consideration of intensity coherence (i.e. minimizing the intensity difference),

$$
\begin{aligned}
\tilde{\alpha}, \tilde{\beta} & =\underset{\substack{\alpha \in[0,1], \beta \in\{0,1\}}}{\arg \min } \int_{\Omega_{\mathcal{L}, Z_{1}}} \underbrace{\alpha \mathcal{E}+(1-\alpha)|\mathcal{E}-\mu|}_{\text {Image term }}+\underbrace{\kappa|\nabla \alpha|}_{\text {Smoothness }}+\underbrace{\beta C_{\mathcal{L}}(\alpha)+(1-\beta) \bar{C}_{\mathcal{L}}(\alpha)}_{\text {Connectivity constraint }} d V \\
\mu & =\int_{\Omega_{\mathcal{L}, Z_{1}}}(1-\alpha) \mathcal{E} d V\left(\int_{\Omega_{\mathcal{L}, Z_{1}}}(1-\alpha) d V\right)^{-1}
\end{aligned}
$$

where $\kappa$ is the smoothness strength which is $0.1 \max (\mathcal{E})$ in our experiment, $\Omega_{\mathcal{L}, Z_{1}}$ is the region within the distance $Z_{1}$ to $\mathcal{L}, C$ is $\infty$ inside the regions $\{\alpha>0.5\}$ isolated from $\mathcal{L}$ and 0 otherwise, $\bar{C}$ is the inverse of $C$ and the final spinal cord segmentation is $\{\tilde{\alpha}>0.5\} \cap\{\tilde{\beta}=1\}$. The minimization of the above functional with respect to $\alpha$ is solved iteratively based on the convex relaxed Potts model [17]. The minimization with respect to $\beta$ is achieved by assigning $\tilde{\beta}=1$ when $C(\tilde{\alpha})=0$. The intensity difference offsets the intensity fluctuation along the structure. Therefore, minimizing the intensity difference well segments a structure along which the intensity is significantly varying.

\section{$3 \quad$ Experiment}

The experiments employ one numerical tensor field (Fig. 2a), three numerical volumes (Fig. 3 a-c), 10 clinical T1-MR and 15 clinical T2-MR images captured from 
25 subjects concerning the cervical and upper thoracic spines 4 . Various components of the proposed method, including the gradient competition descriptor, orientation coherence anisotropy centerline extraction and intensity coherence segmentation are examined. They are compared against five existing approaches using different criteria - the gradient competition descriptor is compared with the multiscale Vesselness measure (Vesselness) [1] and Optimally Oriented Flux approach (OOF) [6] based on curvilinear object orientation estimation accuracy 5 ; the orientation coherence anisotropy centerline extract is studied along with the vesselness measure [1] incorporated in the fast marching method [18] (VesselnessFM) 6 and a state-of-the-art 4D fast marching method (4D-Anisotropy) [4] based on centerline extraction accuracy; and intensity coherence segmentation and the multiphase convex relaxed optimization [19] of Chan-Vese functional (Convex$\mathrm{CV}) 7$ [20] are examined using spinal cord segmentation accuracy.

The first experiment (Fig. 2a) mimics a tensor field computed according to the outer product of the orientation fields of two adjacent tubes. The red region represents the target tube for centerline extraction while the green region corresponds to a high contrast tube (green tensors have a 50\% larger trace than the red counterparts) attached to the target. The vertical red tensors mimic the erroneously detected object orientation, which is perpendicular to the target tube. Assigning this stick tensor field to $\hat{v} \hat{v}^{T}$ in Equation 11 with $\sigma=0$, i.e. orientation coherence is unconsidered, the resultant optimal path is attracted by the green tensors and leaves the target object, giving an incorrect centerline extraction. Fig. 2b shows the tensor field $\mathbf{M}$ obtained by using the tensor field in Fig. 2a for $\hat{v} \hat{v}^{T}$ and $\sigma=1$ voxel-length in Equation[11. The erroneously oriented tensors in Fig. 2a become elliptical tensors in Fig. 2b. Employing the orientation coherence allows the optimal path to pass through these tensors with lower orientation preference and reports a correct extraction.

${ }^{4}$ The scans were acquired by Siemens Avanto 1.5T MRI at St. Joseph's Hospital, London, Canada, using the following protocol: voxelsize $3.3 \times 0.5729 \times 0.5729 \mathrm{~mm}^{3}$ (T1$\mathrm{MR}), 0.4375 \times 0.4375 \times 1 \mathrm{~mm}^{3}(\mathrm{~T} 2-\mathrm{MR})$; slice dimension $640 \times 640 ; T_{R}=2500, T_{E}=$ 29 (T1-MR), $T_{R}=1500, T_{E}=150$ (T2-MR); flip angle $150^{\circ}$. The University of Western Ontario Research Ethics Board for Health Sciences Research Involving Human Subjects approved the use of the clinical data with the requirement for informed consent being waived.

5 The structure orientations of Vesselness and OOF are the eigenvectors of the minimal magnitude eigenvalues of the Hessian matrices and OOF tensors at the selected scales.

${ }^{6}$ The fast marching method finds the optimal path which connects $\boldsymbol{p}_{0}$ and $\boldsymbol{p}_{1}$ to minimize the sum of the inverted vesselness response along the path.

${ }^{7}$ Each spinal image is partitioned into three regions, which correspond to the bright, grey and dark structures, arg $\min _{\left(\alpha_{1}, \alpha_{2}, \alpha_{3}\right)} \sum_{n=\{1,2,3\}} \int \alpha_{n}\left(I-\mu_{n}\right)^{2}+\alpha_{n} H_{n}+$ $\kappa\left|\nabla \alpha_{n}\right| d V, \mu_{n}=\int \alpha_{n} I d V\left(\int \alpha_{n} d V\right)^{-1}$ and $\mu_{1}<\mu_{2}<\mu_{3}, \sum_{n=\{1,2,3\}} \alpha_{n}=1$. The voxels where $\mathcal{L}$ traverses are employed as the hard constraint, i.e. $H_{1}$ and $H_{3}$ are zero except $H_{1}(\mathcal{L})=H_{3}(\mathcal{L})=\infty$, and $H_{2}$ is always zero. Connectivity analysis is performed on the resultant region $\left\{\left\{\alpha_{2}>\alpha_{1}\right\} \cap\left\{\alpha_{2}>\alpha_{3}\right\}\right\}$. The final segmentation is the connected component which encloses $\mathcal{L}$. 
Table 1. The acute angular error (radian) of the structure orientation estimated at the ground truth structure centerline

\begin{tabular}{|c|c|c|c|c|}
\cline { 2 - 5 } \multicolumn{1}{c|}{} & Major radius & 8 & 6 & 4 \\
\hline Structure & Proposed method & $0.1019 \pm 0.0923$ & $0.0774 \pm 0.0337$ & $0.0802 \pm 0.0309$ \\
Orientation & Vesselness & $0.2485 \pm 0.1725$ & $0.2281 \pm 0.1272$ & $0.1852 \pm 0.1104$ \\
& OOF & $0.3178 \pm 0.2045$ & $0.1928 \pm 0.1336$ & $0.1167 \pm 0.0968$ \\
\hline Major axis & Proposed method & $0.1222 \pm 0.1341$ & $0.1210 \pm 0.0864$ & Not Available \\
\hline Minor axis & Proposed method & $0.0525 \pm 0.0364$ & $0.1055 \pm 0.0932$ & Not Available \\
\hline
\end{tabular}

Table 2. Quantitative centerline extraction comparison. An extraction is regarded as successful if the entire extracted centerline stays within the ground truth spinal cord. "Success rate" is the successful proportion of cases among all cases.

\begin{tabular}{|c|c|c||c|c|}
\cline { 2 - 5 } \multicolumn{1}{c|}{} & \multicolumn{2}{c||}{ T1 Images } & \multicolumn{2}{c|}{ T2 Images } \\
\cline { 2 - 5 } \multicolumn{1}{c|}{} & Average distance & Success rate & Average distance & Success rate \\
\hline Proposed method & $1.1853 \pm 0.7831 \mathrm{~mm}$ & $100 \%$ & $0.7895 \pm 0.4536 \mathrm{~mm}$ & $100 \%$ \\
\hline Proposed method & $1.9543 \pm 1.0565 \mathrm{~mm}$ & $100 \%$ & $1.4213 \pm 0.8108 \mathrm{~mm}$ & $100 \%$ \\
without curvilinearity & $3.9686 \pm 2.8130 \mathrm{~mm}$ & $20 \%$ & $1.9087 \pm 1.3180 \mathrm{~mm}$ & $73.33 \%$ \\
Vesselness-FM & $4.5478 \pm 3.3011 \mathrm{~mm}$ & $0 \%$ & $1.4456 \pm 1.1852 \mathrm{~mm}$ & $100 \%$ \\
4D-Anisotropy & \multicolumn{2}{c|}{} & \multicolumn{2}{c|}{}
\end{tabular}

Table 3. Quantitative segmentation results (Mean \pm Standard Deviation)

\begin{tabular}{|c|c|c|c|}
\hline T1 Images & DSC & Signed distance & Absolute distance \\
\hline Proposed method & $0.8156 \pm 0.0786$ & $-0.1650 \pm 0.5863 \mathrm{~mm}$ & $0.9645 \pm 0.4156 \mathrm{~mm}$ \\
\hline Proposed method (Ellipsoid) & $0.7057 \pm 0.1439$ & $-0.7079 \pm 0.9854 \mathrm{~mm}$ & $1.2788 \pm 0.6675 \mathrm{~mm}$ \\
Multiphase CV-Functional & $0.7132 \pm 0.1733$ & $-0.4087 \pm 0.9018 \mathrm{~mm}$ & $1.0087 \pm 0.5854 \mathrm{~mm}$ \\
\hline \hline T2 Images & DSC & Signed distance & Absolute distance \\
\hline Proposed method & $0.8905 \pm 0.0299$ & $-0.2521 \pm 0.3663 \mathrm{~mm}$ & $0.4812 \pm 0.2567 \mathrm{~mm}$ \\
\hline Proposed method (Ellipsoid) & $0.7214 \pm 0.1987$ & $-0.6915 \pm 0.5978 \mathrm{~mm}$ & $1.1795 \pm 0.7109 \mathrm{~mm}$ \\
Multiphase CV-Functional & $0.8201 \pm 0.1535$ & $0.4520 \pm 0.9705 \mathrm{~mm}$ & $0.9087 \pm 0.7018 \mathrm{~mm}$ \\
\hline
\end{tabular}

The second test employs the numerical volumes which consist of nested elliptical and circular curvilinear objects. The target elliptical curvilinear structure is enclosed by another one, and is also adjacent to nested circular curvilinear objects. These neighboring structures exhibit a higher boundary contrast than the target structure (Fig. 3a). Table 1 details the accuracy of estimating different directions of the elliptical object. The proposed method outperforms existing approaches in terms of the structure orientation estimation. Mathematically, Vesselness and OOF merely select one object scale at each position. This is insufficient to capture the elliptical object which has two scales at each centerline position. In contrast, $\lambda_{1}$ and $\lambda_{2}$ of the proposed method correspond to two detection scales. The proposed method has larger performance advantages over existing approaches when the eccentricity of the elliptical structure soars. Without eccentricity (Table 1, major radius=4), the proposed method still yields a better result because of the gradient competition descriptor (Equations 3 and 6). The competition suppresses the detection responses obtained using oversize scales and effectively removes the neighboring disturbance during detection. On the contrary, OOF doesn't have the gradient competition and Vesselness relies on the second derivatives of Gaussian which involves large regions around the target during detection. Their detection performance is unsatisfactory when 


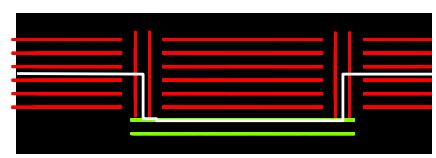

(a)

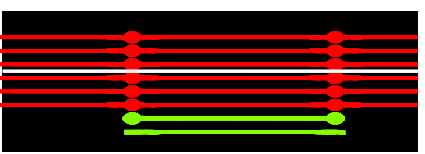

(b)

Fig. 2. The white curves are the optimal paths extracted from the corresponding tensor fields, which are shown as the red or green lines and ellipses. $\boldsymbol{p}_{0}$ and $\boldsymbol{p}_{1}$ are at the center points at the left-most and right-most slices. For better illustration, the tensors with very small traces are not shown.

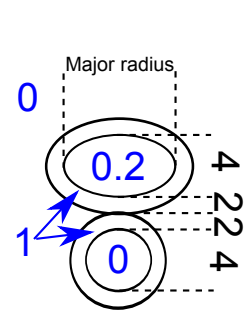

(a)

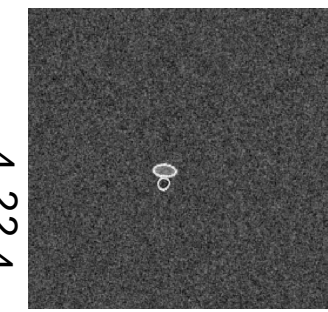

(b)

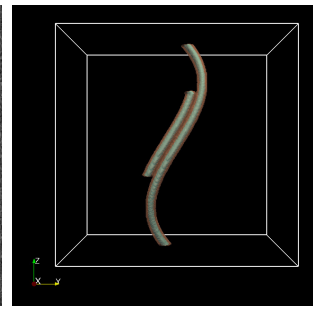

(c)

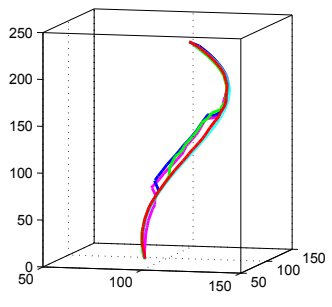

(d)

- Proposed method - Proposed method without orientation coherence - Ground truth — 4D-Anisotropy — Vesselness-FM

Fig. 3. (a) The description of three numerical volumes, major radius $=4,6,8$. The blue and black numbers indicate the intensity of different image regions and the dimensions of different structures respectively. The image is corrupted by additive Gaussian noise with magnitude 0.2. (b-d) Major radius $=8$, (b) the middle axial slice of the volume; (c) the $\mathrm{x}-\mathrm{z}$ view of the isosurfaces of the curvilinear objects with isovalues 0.4 (green) and 0.9 (orange); (d) the centerline extraction results.

strong neighboring disturbance exists even if the target is circular. Finally, Table 1 also evidences the accurate minor and major axes estimated by the gradient competition descriptor of the proposed method.

We further investigate the impact of the neighboring disturbance during centerline extraction. It is noted that the circular tube has a higher boundary contrast than the target object (1 versus 0.8 , Fig. 33). It thus produces larger responses that undesirably attract the optimal paths of Vesselness-FM and 4DAnisotropy to pass through the circular tube (Fig. 3 d). Without employing the orientation coherence (i.e. $\sigma=0$ for Equations 9, 11), the proposed method also fails to keep the optimal path inside the target structure. Analogous results are obtained from the clinical data (Fig. 4). They resemble the discovery in the previous experiment using the numerical tensor field.

Based on the clinical data, the quantitative centerline extraction result is shown in Table 2. The proposed method shows an outstanding performance as compared to other approaches. The low success rates of Vesselness-FM and 4D-Anisotropy hint that these two methods are sensitive to the high contrast tissues adjacent to the spinal cord. In addition, the proposed method is tested 

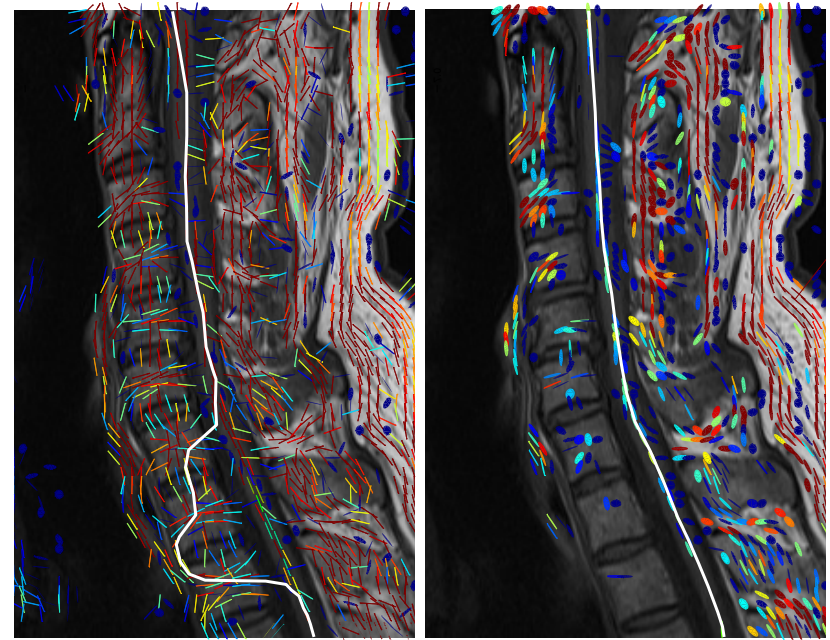

Fig. 4. The extracted centerline based on Equation 10 without (left), and with (right) local orientation coherence. The white curves are the extracted optimal paths. The color lines and ellipses represent the stick tensor $\hat{v} \hat{v}^{T}$ (left) and the tensor $\mathbf{M}$ (right). Their orientation and eccentricity correspond to the directions and anisotropy of these tensors. The tensors are colored according to the largest eigenvalues, pure blue and red are the minimum and maximum value in the image volume respectively. For better illustration, isotropic tensors and tensors having very small traces are not shown.

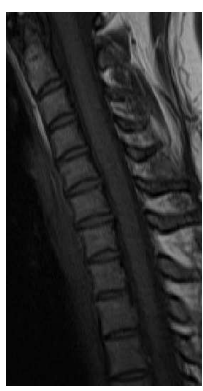

(a)

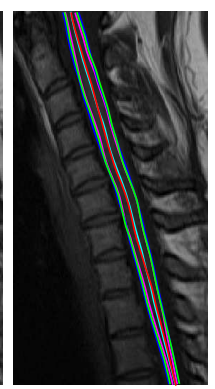

(b)

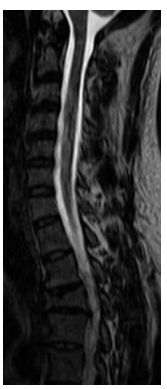

(c)

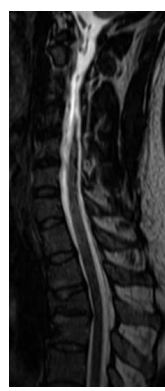

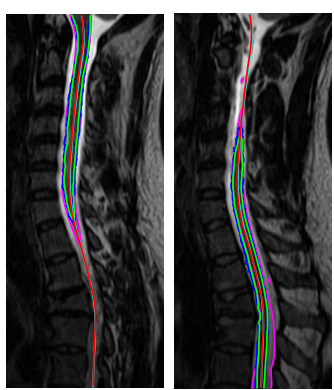

(d)

- Centerline of the proposed method - Segmentation of the proposed method - Ground truth centerline - Segmentation of Convex-CV

- Ground truth segmentation

Fig. 5. Sagittal slices and corresponding segmentation results of 2 clinical cases. (a, b) The mid-sagittal slice of a T1 case. (c, d) Two sagittal slices of a T2 case.

with the curvilinearity measure removed, i.e. $\gamma$ is removed from Equation 11 Nonetheless, the optimal path follows the spinal cord and offers a $100 \%$ success rate, despite the less accurate extraction. This mirrors that the orientation coherence anisotropy offers the robustness against neighboring disturbance, while the curvilinearity helps providing an accurate extraction. 
The spinal cord segmentation results are shown in Table 3 . The proposed method is more accurate than Convex-CV. It is noted that the voxel intensity at $\mathbf{p}_{0}$ are on average $32.28 \%$ and $60.72 \%$ higher than that at $\mathbf{p}_{1}$ in our T1- and T2-MR images respectively. Such significant intensity changes along the spinal cord boosts the Chan-Vese functional and deteriorates the segmentation. In the proposed method, the discrepancy is offset by measuring the intensity difference between reference voxels to the closest centerline positions (Equation 16). The envelop of the ellipsoids constructed according to $\mathcal{L}, r_{1}, r_{2}, \hat{a}_{1, r_{1}}$ and $\hat{a}_{2, r_{2}}$ is also compared to the ground truth spinal cord. It illustrates that the gradient competition descriptor satisfactorily estimates the local ellipsoids that well approximate the spinal cord. Fig. [5 visualizes the segmentation results obtained by the proposed method and Convex-CV. The largely overlapping curves between the proposed method and the ground truth confirms the promising segmentation accuracy of the proposed method.

\section{Perspective and Conclusion}

This study proposes the gradient competition anisotropy for spinal cord centerline extraction and segmentation in T1- and T2-MR images. In the proposed method, the gradient competition descriptor provides an accurate elliptical curvilinear structure detection which is robust against neighboring disturbance. The curvilinearity and orientation field are used to formulate the tensorial orientation coherence anisotropy metric. Despite the high contrast neighboring objects which produce large curvilinearity, the metric encourages the optimal path traversing the target object, and accurately extracts the spinal cord centerlines. The centerline based intensity coherence segmentation model eliminates intensity discrepancy along the structure to perform accurate segmentation.

In the comparison against existing approaches, the proposed method is noticeably more accurate in terms of structure orientation estimation, centerline extraction and structure segmentation. The proposed method merely requires the minimum and maximum scales of the detection target. It is therefore general to handle different elliptical curvilinear objects under strong neighboring disturbance. From this perspective, our experiment compares the proposed method against closely related general curvilinear structure detection methods, including the widely utilized vesselness measure [1], the improved variant of flux measure [6] and the state-of-the-art 4D fast marching algorithm [4]. Applying the proposed method to other curvilinear structure detection applications, such as coronary artery and carotid artery detection will be one of the future directions

${ }^{8}$ DSC (Dice Similarity Coefficient) is computed as $\frac{2|A \cap M|}{|A|+|M|}$ where $A$ and $M$ are the segmented regions of different methods and ground truth respectively. The distance between the segmented regions and ground truth is computed as, at each point on the segmented surface, the Euclidean distance between that point to the closest one on the ground truth. Four samples are drawn for each voxel-length. In the signed distance calculation, negative and positive numbers represent under-segmentation and over-segmentation respectively. 
of this research. Finally, the spinal cord segmentation allows retrieval of clinically relevant information, such as the spinal cord cross-sectional area, spinal cord volume and intensity profiles. Studying the segmentation performance of the proposed method, and extracting disease-specific statistics from the segmented regions based on a large dataset comprising categorized abnormal spines is the current research direction.

Acknowledgement. This work was supported by GE Healthcare and Mitacs through the Mitacs-Elevate fellowship granted to the first author of this paper.

\section{References}

1. Frangi, A.F., Niessen, W.J., Vincken, K.L., Viergever, M.A.: Multiscale vessel enhancement filtering. In: Wells, W.M., Colchester, A.C.F., Delp, S.L. (eds.) MICCAI 1998. LNCS, vol. 1496, pp. 130-137. Springer, Heidelberg (1998)

2. Law, M.W.K., Chung, A.C.S.: An oriented flux symmetry based active contour model for three dimensional vessel segmentation. In: Daniilidis, K., Maragos, P., Paragios, N. (eds.) ECCV 2010, Part III. LNCS, vol. 6313, pp. 720-734. Springer, Heidelberg (2010)

3. Olabarriaga, S.D., Breeuwer, M., Niessen, W.J.: Minimum cost path algorithm for coronary artery central axis tracking in CT images. In: Ellis, R.E., Peters, T.M. (eds.) MICCAI 2003. LNCS, vol. 2879, pp. 687-694. Springer, Heidelberg (2003)

4. Benmansour, F., Cohen, L.: Tubular structure segmentation based on minimal path method and anisotropic enhancement. IJCV 92(2), 192-210 (2011)

5. Bouix, S., Siddiqi, K., Tannenbaum, A.: Flux driven automatic centerline extraction. MedIA 9(3), 209-221 (2005)

6. Law, M.W.K., Chung, A.C.S.: Three dimensional curvilinear structure detection using optimally oriented flux. In: Forsyth, D., Torr, P., Zisserman, A. (eds.) ECCV 2008, Part IV. LNCS, vol. 5305, pp. 368-382. Springer, Heidelberg (2008)

7. Law, M.W.K., Chung, A.C.S.: Efficient implementation for spherical flux computation and its application to vascular segmentation. TIP 18(3), 596-612 (2009)

8. Wörz, S., Rohr, K.: Segmentation and quantification of human vessels using a 3-D cylindrical intensity model. TMI 16(8), 1994-2004 (2007)

9. Law, M.W.K., Chung, A.C.S.: A deformable surface model for vascular segmentation. In: Yang, G.-Z., Hawkes, D., Rueckert, D., Noble, A., Taylor, C. (eds.) MICCAI 2009, Part II. LNCS, vol. 5762, pp. 59-67. Springer, Heidelberg (2009)

10. Koh, J., Kim, T., Chaudhary, V., Dhillon, G.: Automatic segmentation of the spinal cord and the dural sac in lumbar MR images using gradient vector flow field. In: IEEE EMBS, pp. 3117-3120 (2010)

11. Chen, M., Carass, A., Cuzzocreo, J., Bazin, P.L., Reich, D., Prince, J.L.: Topology preserving automatic segmentation of the spinal cord in magnetic resonance images. In: IEEE ISBI. From Nano to Macro., pp. 1737-1740 (2011)

12. McIntosh, C., Hamarneh, G.: Spinal crawlers: Deformable organisms for spinal cord segmentation and analysis. In: Larsen, R., Nielsen, M., Sporring, J. (eds.) MICCAI 2006. LNCS, vol. 4190, pp. 808-815. Springer, Heidelberg (2006)

13. Horsfield, M., Sala, S., Neema, M., Absinta, M., Bakshi, A., Sormani, M., Rocca, M., Bakshi, R., Filippi, M.: Rapid semi-automatic segmentation of the spinal cord from magnetic resonance images: Application in multiple sclerosis. NeuroImage 50(2), 446-455 (2010) 
14. Coulon, O., Hickman, S., Parker, G., Barker, G., Miller, D., Arridge, S.: Quantification of spinal cord atrophy from magnetic resonance images via a b-spline active surface model. MRM 47(6), 1176-1185 (2002)

15. Law, M.W.K., Tay, K., Leung, A., Garvin, G.J., Li, S.: Dilated divergence based scale-space representation for curve analysis. In: Fitzgibbon, A., Lazebnik, S., Perona, P., Sato, Y., Schmid, C. (eds.) ECCV 2012, Part II. LNCS, vol. 7573, pp. 557-571. Springer, Heidelberg (2012)

16. Mirebeau, J.M.: Anisotropic fast-marching on cartesian grids using lattice basis reduction (2012) (Preprint)

17. Chan, T., Esedoglu, S., Nikolova, M.: Algorithms for finding global minimizers of image segmentation and denoising models. SIAM J. App. Math. 66(5), 1632-1648 (2006)

18. Hassouna, M., Farag, A.: Multistencils fast marching methods: A highly accurate solution to the eikonal equation on cartesian domains. PAMI 29(9), 1563-1574 (2007)

19. Bae, E., Yuan, J., Tai, X.C.: Global minimization for continuous multiphase partitioning problems using a dual approach. IJCV 92(1), 112-129 (2011)

20. Chan, T., Vese, L.: Active contours without edges. TIP 10(2), 266-277 (2001) 